\title{
Constraining magnetic field strength in radio relics
}

\author{
A. Botteon ${ }^{* 1,2}$, G. Brunetti ${ }^{2}$ and D. Dallacasa ${ }^{1,2}$ \\ ${ }^{1}$ Dipartimento di Fisica e Astronomia, Università di Bologna, via C. Ranzani 1, 40127 Bologna, \\ Italy \\ ${ }^{2}$ ORA-INAF, via P. Gobetti 101, 40129 Bologna, Italy \\ E-mail: botteoneira.inaf.it
}

Radio relics are diffuse synchrotron sources generally located in the peripheries of galaxy clusters in merging state. According to the current leading scenario, relics trace gigantic cosmological shock waves that cross the intra-cluster medium where particle acceleration occurs. Under the assumption that particles are accelerated at the shock front and are subsequently deposited and then age downstream of the shock, the magnetic field strength in radio relics can be constrained by measuring the thickness of the relic at different radio frequencies.

In this paper we adopt this methodology to the relics in the clusters A521 and CIZA $\mathrm{J} 2242.8+5301$. In these relics we find that a pure radiative aging of electrons downstream is consistent with observations allowing us to constrain the magnetic field strength. These results, together with the lower limits to the magnetic field that we derive from the lack of inverse Compton X-ray emission from the sources, have been combined with Faraday rotation measurements of the Coma cluster to derive a global result. Results suggest that the spatial profile of the magnetic field energy density is broader than that of the thermal gas, implying that the ratio between the thermal and the magnetic field energy densities decreases with cluster radius. Alternatively, radio relics could trace dynamically active regions where the magnetic field strength is increased with respect to the average value in the cluster volume.

EXTRA-RADSUR2015 (*)

20-23 October 2015

Bologna, Italy

(*) This conference has been organized with the support of the Ministry of Foreign Affairs and International Cooperation, Directorate General for the Country Promotion (Bilateral Grant Agreement ZA14GR02 - Mapping the Universe on the Pathway to SKA)

\footnotetext{
* Speaker.
} 


\section{Introduction}

Giant radio relics are synchrotron sources characterized by elongated morphologies and peripheral locations that are found in merging galaxy clusters. There is broad consensus that radio relics trace enormous shocks where the emitting particles can be accelerated or re-accelerated [1]. After being accelerated/re-accelerated, relativistic electrons are then advected into the downstream region at the downstream velocity $V_{d}$ and cool due to inverse Compton (IC) and synchrotron losses

$$
\frac{d \gamma}{d t} \propto-\left(B^{2}+B_{C M B}^{2}\right) \gamma^{2}
$$

where $B$ is the magnetic field and $B_{C M B}=3.25(1+z) \mu \mathrm{G}$ is the equivalent magnetic field due to IC scattering of cosmic microwave background (CMB) photons. The combination of transport and particles aging sets the thickness of radio relics as seen at different frequencies. Emitting electrons can travel a maximum distance from the shock $=V_{d} \tau_{v}$, where $\tau_{v}$ is the particle life-time. For a synchrotron source emitting at redshift $z$ at observing frequency $v$, it is:

$$
\tau_{v} \propto \frac{B^{1 / 2}}{B^{2}+B_{C M B}^{2}} \times[v(1+z)]^{-1 / 2} \propto v^{-1 / 2} .
$$

For this reason the measure of the transverse size of relics at several frequencies $v$ constrains the magnetic field strength in these sources, once the downstream velocity $V_{d}=c_{s} \frac{\mathcal{M}^{2}+3}{4 \mathcal{M}}$ is derived from X-ray observations ( $c_{s}$ is the upstream sound speed and $\mathcal{M}$ is the shock Mach number) [2,3].

\section{Aging and geometric models}

The "standard" scenario where electrons age downstream due to constant (in space) synchrotron and IC losses (Eq. 1.1) can be too simplified as several processes can be triggered in the downstream region.

In Fig. 1 we report the scalings of the relic thickness for different aging scenarios: (i) the case of constant synchrotron and IC losses, (ii) a case where electrons are also re-accelerated by second order Fermi mechanisms downstream, (iii) the case of pure aging, where electrons age in a magnetic field that decays exponentially downstream. We highlight that re-acceleration or a decaying magnetic field downstream can produce either steeper or flatter scalings with respect to the "standard" model $\Phi_{v} \propto v^{-1 / 2}$ (Eq. 1.2). These effects are best seen at low frequency.

In the real case projection effects make things more difficult because they increase the observed transverse size of the relic, $\Phi_{v}$. For this reason we use a basic geometric model in which the observed thickness of the relic is contributed by the projection of the "surface" of the shock (that is frequency independent):

$$
\Phi_{v}=L \cos \theta+V_{d} \tau_{v} \sin \theta
$$

where $L$ is the length of the relic. The free-parameter $\theta$, the angle between the shock surface and the plane of the sky, can be constrained by measuring the thickness (i.e. the FWHM) of the relic at several different radio frequencies. 

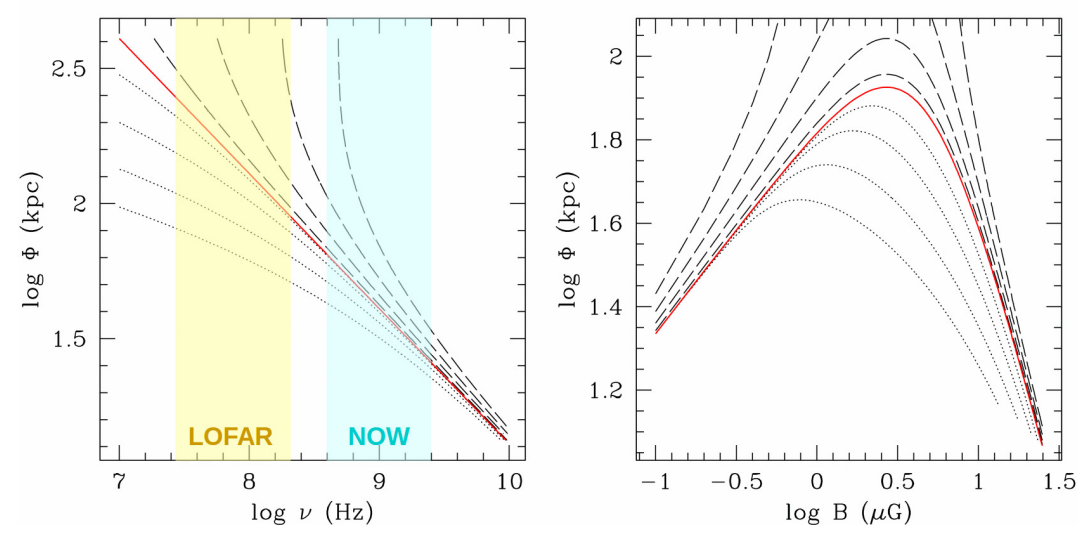

Figure 1: Scalings between the relic thickness at fixed $B=5 \mu \mathrm{G}$ (left) and at fixed $v=327 \mathrm{MHz}$ (right). Red line denotes the "standard" aging (Eq. 1.1). Dashed and dotted lines represent re-acceleration and decaying magnetic field models with different time-scales. The downstream velocity is fixed at $V_{d}=800 \mathrm{~km} \mathrm{~s}^{-1}$.

\section{Magnetic field constraints}

We applied our simple geometric method to the relics in the clusters A $521[4,5]$ and CIZA $\mathrm{J} 2242.8+5301[6,7]$. The availability of X-ray observations allows to obtain the main properties of the underlying shock, for example the downstream velocity. The combination of observations at different radio frequencies allows to fit Eq. (2.1), leading to select a region in the $(B, \theta)$ plane.

The magnetic field estimates must unavoidably bump into the limits due to (i) the lack of IC emission from the relics, which results in a lower limit, $B_{I C}$, to the field strength and (ii) the comparison between thermal $\left(\varepsilon_{t h}=3 n_{e} k_{B} T\right)$ and magnetic $\left(\varepsilon_{B}=B / 8 \pi\right)$ energy densities; with respect to this latter point we assume $\varepsilon_{B}<0.3 \varepsilon_{t h}$.

For the two radio relics results are consistent with a pure radiative aging model (Eq. 1.2), allowing us to readily derive constraints on $B_{d}$ and $\theta$. These are summarized in Tab. 1 together with the limits from IC and thermal/magnetic energy densities.

\begin{tabular}{lcc}
\hline \hline & A 521 & CIZA J2442 $^{\circ}$ \\
\hline$\theta$ & $81^{\circ}-85^{\circ}$ & $83^{\circ}-87^{\circ}$ \\
$B_{d}$ & $0.6-8.5$ & $0.1-14.4$ \\
$B_{d, 0.3}$ & $<7.4$ & $<8.2$ \\
$B_{I C}$ & $>1.2$ & $>1.5$ \\
\hline
\end{tabular}

Table 1: Magnetic field (in $\mu \mathrm{G}$ ) and angle constraints.

We compare our constraints with the magnetic field profile derived with Faraday rotation measurements $(\mathrm{RM})$ for the Coma cluster $[8,9]$. In doing that we re-scale distances in units of $r_{500}$. This combination leads interesting insights about $\varepsilon_{t h} / \varepsilon_{B}$ in galaxy clusters, suggesting that the spatial profile of the magnetic field energy density is broader than that of the thermal gas (Fig. 2). Alternatively, radio relics may trace dynamically active regions where $B$ is locally amplified and then it appears biased high with respect to the average value in the cluster volume; note that indeed also the Faraday RM in [9], at large distances (Fig. 2), are taken in the region of the (Coma) relic. 


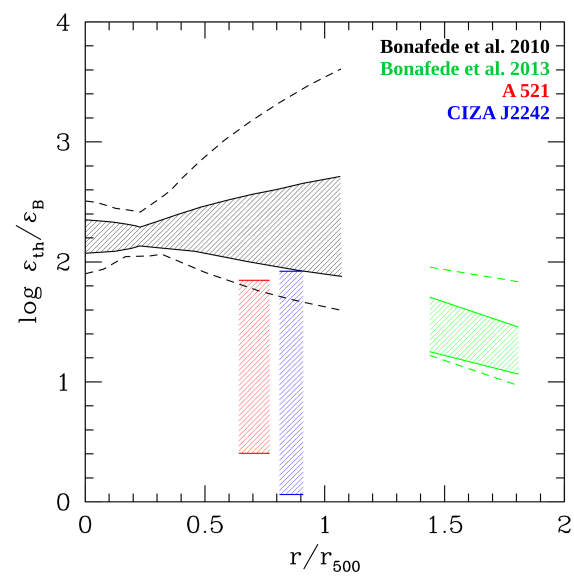

Figure 2: Thermal and magnetic field energy density ratio as a function of $r_{500}$ for three galaxy clusters.

\section{Conclusion and future prospects}

We derive constraints on the magnetic fields in the radio relics in the clusters A 521 and CIZA J2242.8+5301 by modeling the thickness of the relics observed at several frequencies. The limits from the lack of IC emission and from considerations on the magnetic field energy density in the relic region contribute to restrict the allowed field values providing fairly good constraints.

Future radio telescopes, such as LOFAR and SKA, will extend observations at low frequencies allowing to highlight possible additional mechanisms acting in the downstream region (Fig. 1).

\section{References}

[1] G. Brunetti and T. Jones, Cosmic Rays in Galaxy Clusters and Their Nonthermal Emission, Int. J. Mod. Phys. D 23 (2014) 30007, [1401. 7519].

[2] M. Markevitch, F. Govoni, G. Brunetti and D. Jerius, Bow Shock and Radio Halo in the Merging Cluster A520, ApJ 627 (2005) 733-738, [0 412451$].$

[3] R. van Weeren, H. Röttgering, M. Brüggen and M. Hoeft, Particle Acceleration on Megaparsec Scales in a Merging Galaxy Cluster, Science 330 (2010) 347, [1010 . 430 6].

[4] S. Giacintucci, T. Venturi, G. Macario, D. Dallacasa et al., Shock acceleration as origin of the radio relic in A 521?, A\&A 486 (2008) 347-358, [0803.4127].

[5] H. Bourdin, P. Mazzotta, M. Markevitch, S. Giacintucci et al., Shock Heating of the Merging Galaxy Cluster A521, ApJ 764 (2013) 82, [1302.0696].

[6] A. Stroe, R. van Weeren, H. Intema, H. Röttgering et al., Discovery of spectral curvature in the shock downstream region: CIZA J2242.8+5301, A\&A 555 (2013) A110, [1305. 0005 ].

[7] H. Akamatsu and H. Kawahara, Systematic X-Ray Analysis of Radio Relic Clusters with Suzaku, PASJ 65 (2013) 16, [1112.3030].

[8] A. Bonafede, L. Feretti, M. Murgia, F. Govoni et al., The Coma cluster magnetic field from Faraday rotation measures, A\&A 513 (2010) A30, [1 002 . 0594].

[9] A. Bonafede, F. Vazza, M. Brüggen, M. Murgia et al., Measurements and simulation of Faraday rotation across the Coma radio relic, MNRAS 433 (2013) 3208-3226, [1305 . 7228]. 